\title{
LÓGICAS GLOBALES EN LA ORGANIZACIÓN DEL TERRITORIO MADRILEÑO*
}

\author{
Zárate Martín, M. A. \\ Departamento de Geografía de la UNED
}

\begin{abstract}
Resumen: Numerosos indicadores hacen de la región de Madrid uno de los territorios más dinámicos del Mediterráneo. Las rażones de su expansión se encuentran en la adaptación de su economía a las oportunidades de la globalización y en los esfuerzos del gobierno regional por superar desequilibrios heredados, mejorar la calidad de vida de sus habitantes y adecuar el territorio a las exigencias de un sistema internacionalizado. Las lógicas de la globalización son también responsables de las mayores transformaciones paisajísticas experimentadas por la Región madrileña a lo largo de su historia. Asistimos al nacimiento de una modelo territorial que aspira a transformar su estructura radial por otra reticular. El modelo "metropolitano", que contraponía centro y periferia, se sustituye por un modelo de "ciudad-región" difusa y polinuclear, donde adquieren protagonismo las desigualdades ante el empleo, las dificultades de acceso a la vivienda, el envejecimiento poblacional, la inmigración extranjera y los riesgos de exclusión.
\end{abstract}

Palabras clave: Ciudad-región, Estructura reticular, Sistema polinuclear, Calidad de vida, Transformaciones paisajisticas

\begin{abstract}
Numerous indicators do of the region of Madrid one of the most dynamical territories of the Mediterranean. The reasons of his expansion are in the adjustment of his economy to the opportunities of the globalization and in the efforts of the regional government for overcoming inherited imbalances, improving the quality of life of his inhabitants and adapting the territory to the requirements of an internationalized system. The logics of the globalization are also responsible for the major landscape transformations experienced by the region of Madrid along his history. We are present at the birth of a territorial model who aspires to transform his radial structure for reticulated other one. The "metropolitan" model, who was opposing center and periphery, is replaced by a model of diffuse and polynuclear "city-region", where the disparitys acquire protagonism before the employment, the difficulties of access to the housing, the population aging, the foreign immigration and the risks of exclusion.
\end{abstract}

Key words: City-region, Network structure, Polynuclear system, Quality of life, Landscape transformations.

* Recibido: 10-11-03. Aceptado: 4-7-04. 


\section{Introducción}

La mundialización de la economía introduce lógicas en la organización del territorio que modifican las relaciones entre ciudades, la jerarquía urbana y la estructura de las ciudades. Entre las consecuencias espaciales de la globalización figuran la aparición de paisajes urbanos nuevos, la desaparición de los limites entre la ciudad y el campo, y el nacimiento de ejes de diferenciación social que se asocian a la situación personal ante el empleo. La afluencia masiva de inmigrantes, otra consecuencia de la globalización, plantea desafíos de convivencia e integración para una sociedad como la española, tan poco acostumbrada a la presencia masiva de extranjeros en tiempos recientes.

Dentro de España y en el conjunto de Europa, la aglomeración madrileña y su ámbito de influencia más directa, la Comunidad autónoma del mismo nombre y parte de las provincias limítrofes de otras Comunidades, se convierten en un modelo paradigmático de las transformaciones que la globalización provoca en el territorio, en la sociedad y en el comportamiento de los individuos. En nuestro caso, los orígenes de estos cambios se hallan en los factores de oportunidad que representaron la adhesión de España a la Unión Europea y la plena integración funcional de Madrid en el sistema de grandes metrópolis del planeta, como nodo secundario de transmisión y difusión de innovaciones, gracias sobre todo al aeropuerto de Barajas. En los comienzos de un nuevo milenio, la mayoría de los indicadores de desarrollo y crecimiento económico hacen de Madrid uno de los territorios de mayor dinamismo dentro del sistema mundial de ciudades (Córdoba, J. y Gago, C. 2002). Un reciente estudio de la Datar sitúa a Madrid en un segundo rango dentro de la jerarquía urbana de Europa, a la par que Milán, sólo por debajo de Londres y Paris (Rozenblat, C. et Cicille, P. 2003).

En la actualidad, la Comunidad autónoma de Madrid se comporta funcionalmente como una región urbana de $8.028 \mathrm{~km} 2$, bajo la influencia directa de la capital, con una población de casi 5 millones de habitantes, una moderna industria y un sector de servicios muy avanzado. Lo que bien podemos calificar como "ciudad-región" de Madrid es el principal centro de servicios del Estado, produce más del $14 \%$ del valor añadido nacional y en 2003 atrajo el $72 \%$ de las inversiones de capital extranjero en España. Su situación central respecto al conjunto peninsular ha favorecido el crecimiento industrial y el desarrollo de los servicios. El aeropuerto de Barajas y los sistemas radiales de gran velocidad aseguran las relaciones con otras regiones del planeta, garantizan la movilidad de las personas, de los capitales, de las mercancías y de las ideas dentro del proceso general de globalización. Todo favorece, además, el protagonismo de Madrid como ciudad internacional de congresos y ferias, y como primer destino turístico de interior peninsular. El hecho de la capitalidad del Estado, por su parte, genera oportunidades para el desarrollo: mercado interior, amplitud de servi- 
cios, abundancia y formación de la mano de obra, diversificación del tejido industrial y proximidad a los centros de dirección y de toma de decisión.

Especialmente importante como elemento de crecimiento económico y motor de transformación del territorio fue la creación de la autonomía madrileña en 1982. Resulta sorprendente, pero explicable desde planteamientos políticos, económicos y sociales, comprobar como la Comunidad de Madrid ha terminado por ser la más beneficiada indirectamente por la descentralización estatal. El nacimiento de la autonomía ha permitido al gobierno regional concentrar todo su esfuerzo en la modernización de un territorio de reducida superficie y en la mejora del bienestar de sus ciudadanos. A diferencia de lo que sucede en otras Comunidades, algunas con problemas de identidad nacional que comportan elevados costes económicos y sociales, las autoridades madrileñas han podido hacer de la ordenación territorial un tema prioritario y de consenso político, como sus sucesivos presidentes han repetido reiteradamente en actos públicos, discursos institucionales y escritos (Ruíz Gallardón, A.,1999).

Enfrentados a la quiebra del modelo productivo y urbano de la etapa desarrollista, los responsables políticos y los técnicos del planeamiento madrileños iniciaron ya en la década de los 80 estrategias de articulación territorial dirigidas a la expansión de la economía regional y a la mejora de las condiciones de vida de sus habitantes. Desde entonces, las autoridades autonómicas y municipales aprovechan con eficacia los cauces institucionales y de financiación ofrecidos por la Unión Europea y los aspectos positivos de la globalización para dinamizar la economía regional y adaptarla a un sistema productivo cada vez más competitivo a escala mundial.

\section{Lógicas espaciales de los agentes económicos}

En los comienzos del III milenio, las lógicas espaciales de un capital cada vez más multinacional impulsan en todo el mundo, y por supuesto en Madrid, un modelo de ciudad difusa que expande las periferias y transforma los espacios centrales. La globalización y los factores de oportunidad generados por la capitalidad y la autonomía política han sido y siguen siendo determinantes para comprender la fuerte expansión de la actividad económica madrileña durante los últimos años. Una de las principales consecuencias de este dinamismo regional ha sido el constante incremento de la demanda de suelo para oficinas, con su consiguiente repercusión sobre los precio del suelo y de la vivienda, que figuran entre los más altos de España.

La afluencia masiva de capital extranjero a partir de los años 80 y la insuficiencia de suelo en el municipio madrileño para usos terciarios contribuyen para hacer de Madrid la octava ciudad más cara de Europa en el sector inmobiliario de oficinas. En 
el año 2000, los precios de alquiler de inmuebles de oficinas subieron un $50 \%$ para el conjunto de la ciudad respecto a 1999. En el 2001, el metro cuadrado de oficina costaba el equivalente a 36 euros, muy por encima de los 24 euros pagados aquel año por metro cuadrado para el mismo uso en Barcelona. Desde entonces se ha mantenido la tendencia al aumento de estos precios y, según los expertos de "CB Richard Ellis", las previsiones son de un continuo incremento en el futuro inmediato.

Para hacer frente a la insuficiencia de suelo para oficinas en Madrid, dentro de la almendra que dibuja la M-30, muchas empresas prefieren localizarse en los bordes del municipio y en las nuevas periferias del área metropolitana. Las ventajas de estas localizaciones son manifiestas por el menor precio del suelo, la abundancia de espacio y las facilidades para integrar actividades complementarias de servicios en unas mismas superficies. También atraen la realización de beneficios por las plusvalías que resultan de la venta de antiguas instalaciones, y la concentración de empleados en un solo edificio o en un complejo de edificios, lo que permite reducir los costes de personal. Grandes empresas, como Endesa, Unión Fenosa, Indra, Sogecable o Vodafone, han trasladado sus sedes corporativas a Tres Cantos, Boadilla del Monte, el PAU de las Tablas, el Parque de las Naciones y San Sebastián de los Reyes. La Compañía Telefónica también tiene previsto el traslado de su sede al PAU de las Tablas, después de abandonar 1,2 millones de $\mathrm{m} 2$ en el centro de Madrid, y el Banco Santander Central Hispano está construyendo un "campus" financiero para su uso exclusivo en la localidad de Boadilla del Monte.

Dentro de la almendra central, las actividades terciarias ocupan zonas cada vez más diferenciadas, siguiendo exigencias concretas de localización, factores ambientales, de accesibilidad y de oportunidad. Por otra parte, desde la segunda mitad del siglo XX, continua la sustitución de viviendas por oficinas y comercios dentro del Ensanche decimonónico. El aumento de hoteles en el distrito Centro también está siendo espectacular, por el crecimiento del turismo de negocios hacia Madrid, por el papel de puerta de entrada del aeropuerto de Barajas y por la expansión de un turismo de interior que se dirige hacia la capital interesado por sus atractivos culturales y monumentales, y por el renombre internacional de sus alrededores, especialmente de Toledo (Zárate, M. A., 2002).

El vaciamiento demográfico del centro de Madrid desde 1960 a 1996 ha propiciado la desaparición de su comercio tradicional, sustituido, unas veces, por un comercio banal y comercios mayoristas, y otras, por franquicias de cadenas nacionales y multinacionales, en este último caso, sobre todo, en las calles principales y más frecuentadas. Los comercios en cadena, desde alimentación a perfumería y moda en el vestir, representan la adaptación del comercio interior a los nuevos tiempos; son una de las respuestas del comercio central a las nuevas formas de consumo y a la competencia que plantean en el conjunto de las ciudades las grandes superficies y supermercados que se localizan preferentemente en las periferias residenciales. Ahora bien, 
la mayoría de los comercios y locales de servicios pertenecientes casi siempre a cadenas multinacionales está pensada más para la demanda de la población que se desplaza a diario al centro por razones de trabajo, de negocios, de compra o turismo, que para los propios residentes de los barrios en los que se localiza, integrados en una proporción muy elevada por ancianos y personas de rentas muy bajas.

La instalación masiva de extranjeros dentro de Madrid, uno de los fenómenos más espectaculares de su transformación urbana en los últimos años, sobre todo en ciertos barrios, es otras de las manifestaciones más significativas de unas lógicas espaciales de la globalización que favorecen los desplazamientos a escala planetaria, no sólo de las personas sino también del capital y de las inversiones financieras, incluidas las sumas de dinero que mueven las actividades vinculadas a la inmigración y las divisas que los inmigrantes transfieren a sus países de procedencia. La concentración excesiva del comercio mayorista chino en pleno corazón del barrio de Lavapiés, uno de los más tradicionales y castizos del viejo Madrid, constituye uno de los ejemplos más representativos de cambio funcional, transformación social e, incluso, modificación de paisaje promovido por las nuevas lógicas espaciales en los espacios centrales de nuestras ciudades. En el caso concreto de este barrio madrileño, lo que siempre ha sido un espacio plurifuncional se ha convertido en muy poco tiempo en un espacio monofuncional, en un espacio ocupado casi exclusivamente por almacenes y tiendas de venta de ropa al por mayor que entran en conflicto con las necesidades e intereses de una población autóctona cada vez menos numerosa, que corre el peligro de convertirse en residual (Zárate, M. A., 2003). Poco a poco, la progresiva expansión del comercio chino amenaza con transformar el barrio de Lavapiés en una auténtica "China Town", superando una realidad social actual que lo configura como ámbito multicultural.

Por otra parte, las lógicas espaciales de la globalización son también responsables de la transformaciones más recientes de los paisajes industriales madrileños. En efecto, las exigencias del modelo de producción postfordista, inherentes a la mundialización de la economía, han dado origen a nuevos paisajes industriales. La fragmentación de la producción, la complementariedad empresarial, la demanda creciente de servicios para la industria y consecuentemente el aumento de los trabajadores de cuello blanco sobre los de mono azul, propician la mezcla de usos del suelo en los parques industriales de última generación que han proliferado por los municipios de la región. Muchos de ellos, calificados como parques empresariales mixtos, combinan actividades industriales y terciarias, casi siempre complementarias. Todos estos asentamientos disponen de medios centralizados de seguridad y de gestión, de asistencia jurídica y estudios de mercado, de depósitos de mercancías y buenas comunicaciones, lo que resulta importante cuando se trata de actividades internacionalizadas, muy numerosas en el caso madrileño.

Las tipologías más innovadoras de polígonos industriales de última generación y de parques empresariales mixtos se hacen patentes en numerosas localidades, pero 
sobre todo en Torrejón de Ardoz, San Fernando de Henares, Arganda del Rey, Arroyo Culebro y Cerro de la Cantueña (Fuenlabrada). Precisamente, el Arroyo Culebro y el Cerro de la Cantueña constituyen las piezas esenciales de una de las operaciones más ambiciosas y de resultados más espectaculares en la transformación del territorio madrileño, la incluida dentro de la denominada Estrategia Global del Gran Sur metropolitano, que el gobierno regional de Madrid puso en marcha en 1989. El éxito de esta estrategia como instrumento de creación de tejido urbano se ha puesto de manifiesto con la inauguración de Metrosur, el 11 de abril de 2003, que, a su vez, evidencia la fuerte expansión residencial y de empleo durante la última década de las localidades de Alcorcón, Móstoles, Fuenlabrada, Getafe y Leganés.

La internacionalización del aparato productivo madrileño, la expansión de las nuevas tecnologías, las facilidades de acceso a los estudios superiores y, en definitiva, el auge de la denominada "revolución del conocimiento" explican la creación de nuevas universidades, públicas y privadas, y la habilitación de espacios para actividades de $I+D$ en sus inmediaciones. Los parques científicos o polos tecnológicos próximos a las nuevas universidades se han convertido en uno de los principales instrumentos de la administración para reequilibrar el territorio regional y propiciar el desarrollo de las zonas que fueron más afectadas por la crisis industrial de los años 70 . Con ambas medidas, con la puesta en marcha de nuevas universidades y la creación de parques científicos, generalmente asociados a ellas, se está impulsando el desarrollo de las localidades del sur y del este madrileños, haciéndolas dinámicas, atractivas para la actividad económica y competitivas desde el punto de vista empresarial, en consonancia con los "Principios de una Política Europea de Desarrollo Territorial", publicados en 1994, y con las directrices de la "Estrategia Territorial Europea", aprobadas en la Conferencia de Postdam (1999).

Actualmente, los "campus" madrileños se hallan en fase de ampliación y todas las universidades disponen de parques científicos asociados. Las dos universidades públicas más recientes, la "Carlos III" y la "Rey Juan Carlos I", en el sur metropolitano, son las que están teniendo mayor repercusión sobre la organización del territorio. Su puesta en servicio ha evitado la concentración del saber en la zona norte y ha venido a reparar uno de los desequilibrios que formaban parte de lo que se entendía como "deuda social" del conjunto de la Comunidad con el sur de la aglomeración madrileña, por su acumulación de carencias en equipamientos e infraestructuras desde los años 60.

Los nuevos "campus" y sus parques científicos se ubican en terrenos de gran accesibilidad, cuentan con servicios de ferrocarril de cercanías y metro, y se hallan siempre en localizaciones próximas o inmediatas a las principales autovías de la red metropolitana. Todas y cada una de estas superficies responden siempre a criterios de calidad urbanística y arquitectónica, la edificación es de baja densidad y los espacios ajardinados, las áreas deportivas y de ocio, son abundantes. Para consolidar y asegurar en el tiempo la eficacia de los efectos dinamizadores del "conocimiento" sobre el 
territorio, la administración ha establecido reservas de suelo para nuevos "campus" en otras localidades, pero dando siempre prioridad a las localizaciones que, según el planeamiento regional vigente, han de reforzar las áreas de más reciente centralidad, como Campamento, Arroyo Culebro, Rivas, Aranjuez y la Hoya de Villalba.

\section{Nuevas lógicas residenciales y espacios de ocio}

La internacionalización de la economía madrileña, la inmigración extranjera y la necesidad de alojamientos para los más jóvenes incrementan constantemente la demanda de suelo residencial en nuestra Comunidad y en las provincias limítrofes. Cada vez más localidades madrileñas y de Toledo y Guadalajara se transforman en "ciudades dormitorios" y "ciudades satélites" de Madrid. No menos importante, entre las razones que explican el imparable aumento de la demanda de viviendas en nuestra región, es el proceso de intensa especulación inmobiliaria que se desarrolla en su interior y del que participan otros españoles, e incluso extranjeros, al desviar en los últimos años parte del ahorro desde los fondos de inversión en bolsa a fondos inmobiliarios o simplemente a la compra de pisos. La consecuencia es un aumento escandaloso y continuo de los precios de la vivienda. La capital se ha convertido en la ciudad española donde la vivienda resulta más cara y donde el acceso a la misma es más difícil, sobre todo para los matrimonios jóvenes y los colectivos de rentas más bajas. En la Comunidad de Madrid, los precios subieron un 23\% en 2002, según datos del propio Ministerio de Fomento.

Para resolver las carencias residenciales, con un horizonte de crecimiento de la población regional hasta el año 2025, el Plan General de Ordenación Urbana de Madrid 1997 y las Bases de Estrategias Territoriales de la Comunidad de 1995 ampliaron muchísimo las previsiones de construcción de viviendas de todo tipo, libres y de precio tasado, introduciendo en el mercado una gran cantidad de suelo urbanizable. La formula preferida para la creación de los espacios residenciales nuevos ha sido la de los ensanches y PAUs (Programas de Actuación Urbanística Urgente), ya iniciada en el caso madrileño en los 80. La administración ha apostado por la construcción de espacios urbanos coherentes y cualificados, bien equipados y sin las deficiencias estructurales de los años 60 y principios de los 70, aunque la calidad en el diseño arquitectónico de las viviendas brilla por su ausencia, si se exceptúan algunos encargos de la Empresa Municipal de la Vivienda, la única que se ha preocupado realmente por desarrollar casas de vanguardia en los nuevos PAUs.

Las lógicas de la globalización son también responsables de los nuevos paisajes residenciales. En los PAUs, se ha impuesto la construcción compacta a partir de la recuperación de la manzana cerrada y de un concepto de ciudad sostenible que se materializa en fórmulas de "naturación" urbana y ahorro energético. Se trata de no 
perder lo que constituye la forma característica de la ciudad mediterránea, en la que los usos del suelo se mezclan dentro de las zonas residenciales (viviendas, oficinas, centros comerciales, dotaciones), y de recuperar la calle, que el "open planning" de la ciudad funcional había hecho desaparecer. Otros elementos esenciales del urbanismo de los PAUs son la preocupación por asegurar las buenas comunicaciones con las áreas centrales de la ciudad y la creación de puntos de encuentro para facilitar las relaciones entre los ciudadanos.

Por otra parte, desde los años 80 , proliferan por toda la región, como en el conjunto de la geografía española, las urbanizaciones de viviendas unifamiliares, si bien sus mayores proporciones respecto al total de las nuevas edificaciones corresponden a los municipios madrileños de mayor crecimiento demográfico entre 1996 y la actualidad (ver mapa 1). En este caso, estas urbanizaciones, de viviendas unifamiliares exentas, pareadas o adosadas, responden fundamentalmente a modelos residenciales y estilos de vida anglosajones que la televisión y el cine difunden a través de la globalización cultural. No obstante, las tipologías morfológicas y las componentes sociales dominantes en estas urbanizaciones son muy variadas; sus elementos comunes son las bajas densidades residenciales, una enorme dependencia del automóvil para garantizar la movilidad personal y comportamientos "rururbanos" que se materializan en una mínima relación con la naturaleza y en hábitos de consumo estrictamente ciudadanos.

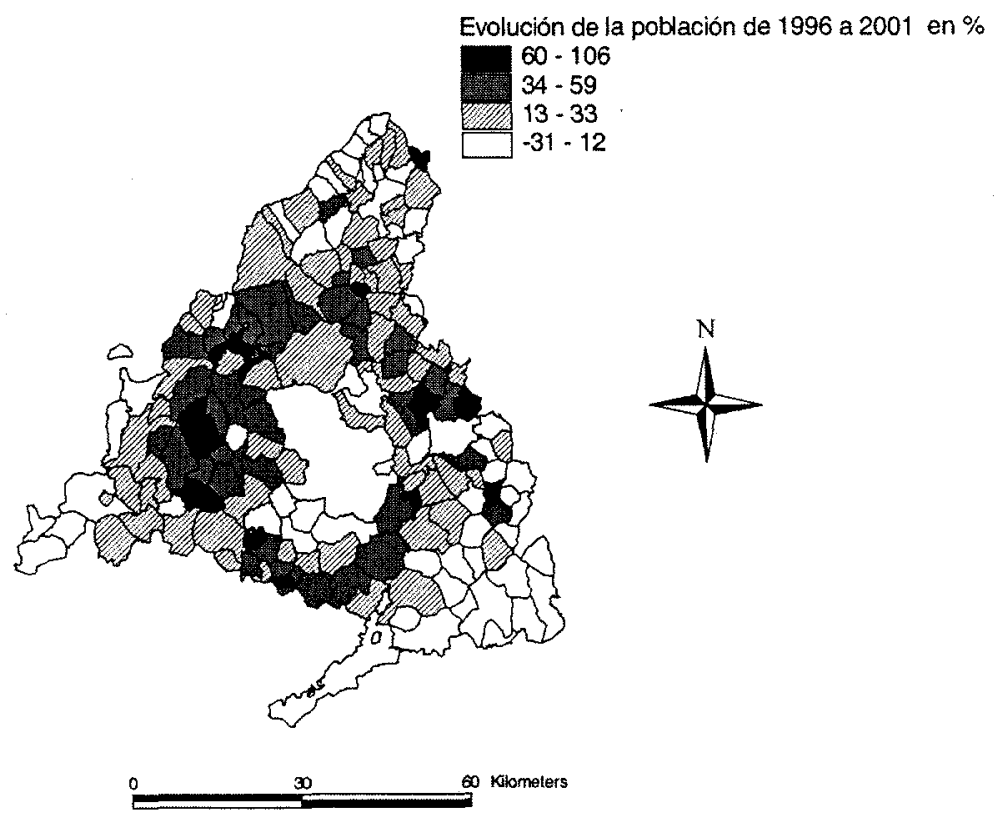

Mapa 1. Evolución de la población en los municipios de la Comunidad de Madrid entre 1996 y 2001. Fuente: Elaboración propia. 
Por último, las dinámicas de la globalización se encuentran detrás de la proliferación de importantes espacios de ocio en la región madrileña. El aumento generalizado del nivel de vida, la forma cada vez más extensa de la ciudad, la asimilación de los modos de entretenimiento característicos de la sociedad norteamericana y las facilidades para la inversión de capital extranjero son factores espontáneos asociados a la globalización que explican la creación de espacios de ocio a lo largo y ancho de la Comunidad, con importantes efectos paisajísticos y de generación de empleo. La administración, consciente de la importancia de estas actividades como factores de desarrollo económico y de su papel en la ordenación del territorio, impulsa también la creación de este tipo de espacios mediante el planeamiento oficial. En este sentido, sobresalen y resultan espectaculares por la superficie afectada, el volumen de las inversiones requeridas, el impacto funcional provocado y las consecuencias paisajisticas, el parque temático de la Warner Bross, en San Martín de la Vega, y el parque de nieve "Xanadú", en Arroyomolinos, junto a la carretera de Extremadura (Zárate, M. A. 2003).

\section{Las lógicas espaciales del poder político}

Las importantes transformaciones del territorio madrileño a las que se viene haciendo referencia, no podrían entenderse sin el papel desempeñado directamente por la administración a través de las posibilidades legales que ofrecen la Constitución y el Estatuto de Autonomía. Desde el nacimiento de la Comunidad autónoma de Madrid, las autoridades regionales y municipales, incluso cuando no coinciden los partidos políticos en el poder, han sabido entenderse para armonizar estrategias dirigidas a sustituir la estructura territorial radial del pasado, totalmente convergente hacia la aglomeración madrileña y una de las causas de sus deseconomías de escala, por otra reticular, capaz de multiplicar las interconexiones entre los principales asentamientos de la región. Los objetivos de esta estrategia son dobles: por una parte, reducir los inconvenientes que se derivan de la excesiva macrocefalia de la capital para el conjunto de la región; por otra, proporcionar oportunidades de desarrollo económico y posibilidades de crecimiento urbanístico a otros municipios, sobre todo a aquellos que se localizan en espacios metropolitanos susceptibles de competir con las regiones europeas más prósperas o que se seleccionan como núcleos de reequilibrio regional.

Para avanzar hacia un desarrollo territorial más equilibrado y sostenible de la región madrileña, el gobierno autónomo ha seleccionados unos municipios que deben actuar como focos de difusión de crecimiento y desarrollo económico (Ver mapa $n^{\circ} 2$ ). Estos municipios coinciden con aquellos que vienen funcionando como cabezas de partidos judiciales desde el siglo XIX y que, por lo tanto, ya eran lugares centrales para núcleos de población de menor rango. Se entiende que la expansión de estos municipios quitará "centralidad al centro" y servirá de base para for- 
mar una red urbana policéntrica, coincidiendo en este sentido con las recomendaciones de la Estrategia Territorial Europea, aprobada en la Conferencia de Postdam (1999). De ese modo se podrán hacer compatibles los objetivos de crecimiento económico, mejora de la calidad de vida de la población y respeto por la naturaleza y el patrimonio cultural.

La preocupación por hacer frente a los problemas de la excesiva macrocefalia de la capital explica también la coordinación de las estrategias regionales y municipales para crear suelos de usos especializados en la periferia de la aglomeración madrileña y en localizaciones más centrales que resultan complementarias o que sufrieron con más intensidad la crisis industrial de los años 70 y parte de los 80 . Dentro de la ciudad de Madrid, esto es lo que sucede en la zona de Campamento, aprovechando el

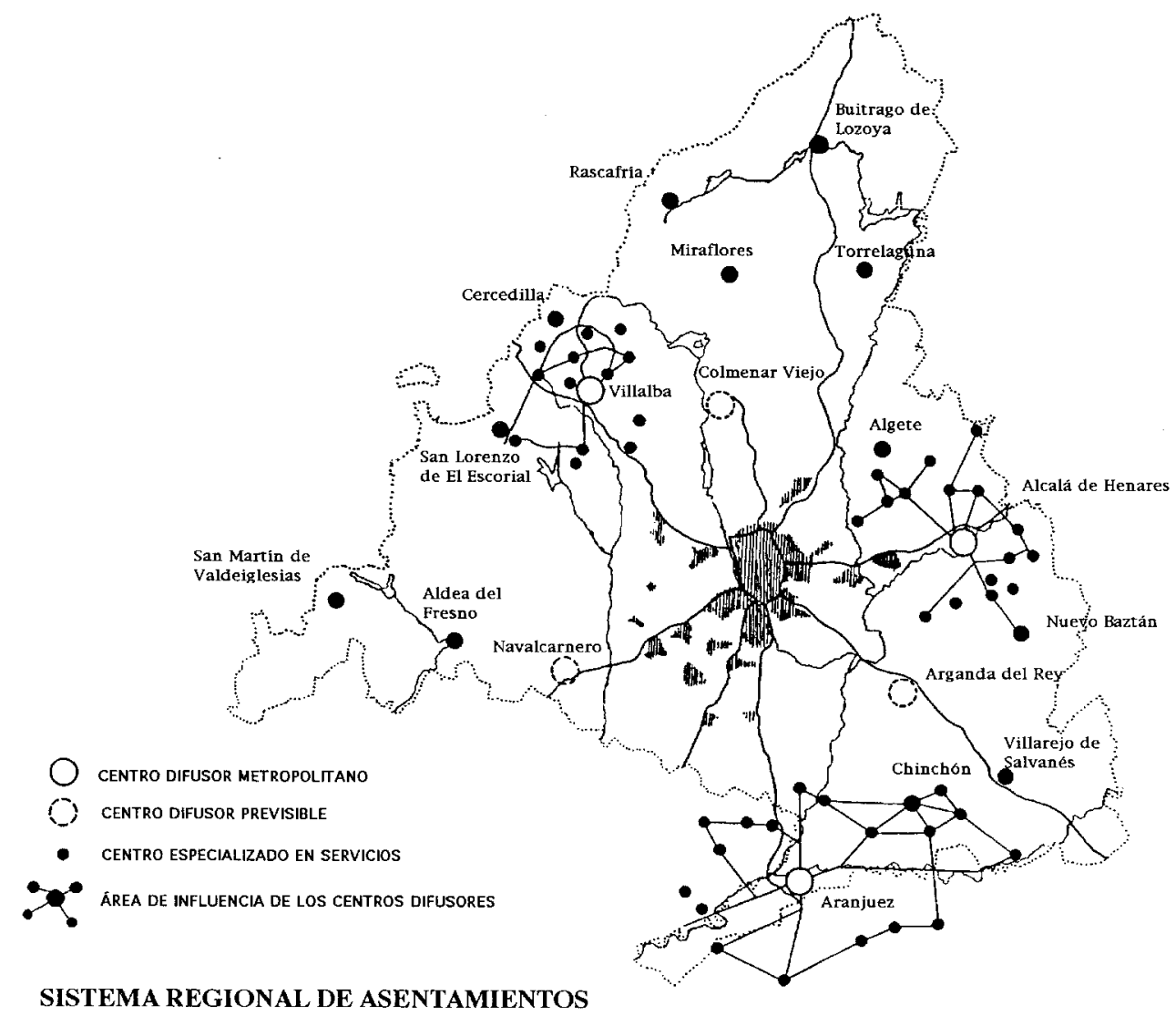

Mapa 2. Focos de difusión y crecimiento previstos en la región madrileña. Fuente: CAM. 
espacio de sus antiguas y numerosas instalaciones militares, y en los distritos de Arganzuela y Villaverde. La recuperación de la escala metropolitana por el Plan General de Ordenación Urbana de Madrid de 1997, aunque limitada al territorio incluido entre las autovías de La Coruña y de Barcelona, en la corona norte del municipio, se está utilizando, así mismo, para impulsar la desconcentración espacial del terciario madrileño.

Los criterios de accesibilidad respecto a las vías de comunicación y de calidad ambiental actúan de manera determinantes para la administración a la hora de decidir la localización de los nuevos parques de oficinas. La mayoría se ubica junto a las vías de circunvalación M-30 y M-40, y en las áreas de conexión de la M-50 con las autovías y autopistas radiales y la red ferroviaria de cercanías, como ocurre con el parque empresarial de Las Rozas. Cerca de Campamento, se han levantado el Parque de Arroyo Meaques, y en el término municipal de Alcorcón, otros importantes parques de oficinas y de actividades terciarias en general. En el norte y sureste de la periferia metropolitana se han habilitado grandes espacios para actividades terciarias, destacando los correspondientes a dos de los polos propuestos como centros difusores regionales de desarrollo: Alcobendas-San Sebastián de los Reyes, por un lado, y Arganda del Rey-Rivas Vaciamadrid, por otro. También sobresalen por las inversiones realizadas los parques de actividad de Alcalá de Henares y del eje del Arroyo Culebro, concebidos como polígonos mixtos, con suelo para industrias y oficinas.

Cuando se trata de las grandes superficies comerciales, su ubicación concreta es el resultado de una decisión conjunta entre las autoridades locales y regionales a través del planeamiento municipal y de los Planes de Acción Comercial (PAC) que desarrolla la "Dirección General de Consumo de la Comunidad de Madrid". Las decisiones se toman después de analizar la estructura comercial de cada zona, una vez identificadas las carencias respecto a la clientela potencial y valorados los desplazamientos que los usuarios han de efectuar para satisfacer sus demandas. Muy a menudo, las actividades comerciales complementan actividades de oficinas en unos mismos emplazamientos, como sucede en los parques empresariales de Las Rozas y de Alcorcón. Los Centros de Transporte de Getafe y Coslada y el Centro de Servicios de Producción del Arroyo Culebro, diseñados por el gobierno regional en 1987, disponen actualmente de servicios avanzados para las empresas y de comercios para las personas que trabajan en estos complejos y en las zonas industriales próximas.

Finalmente, la administración regional fue también responsable de la creación y localización de los polos tecnológicos para industrias de nueva generación de Tres Cantos, Las Rozas y el Corredor del Henares, en el marco de las políticas de reindustrialización que se acometieron en los años 80 . En todos estos casos, se buscó la proximidad a universidades y la existencia de conexiones fáciles con el aeropuerto 


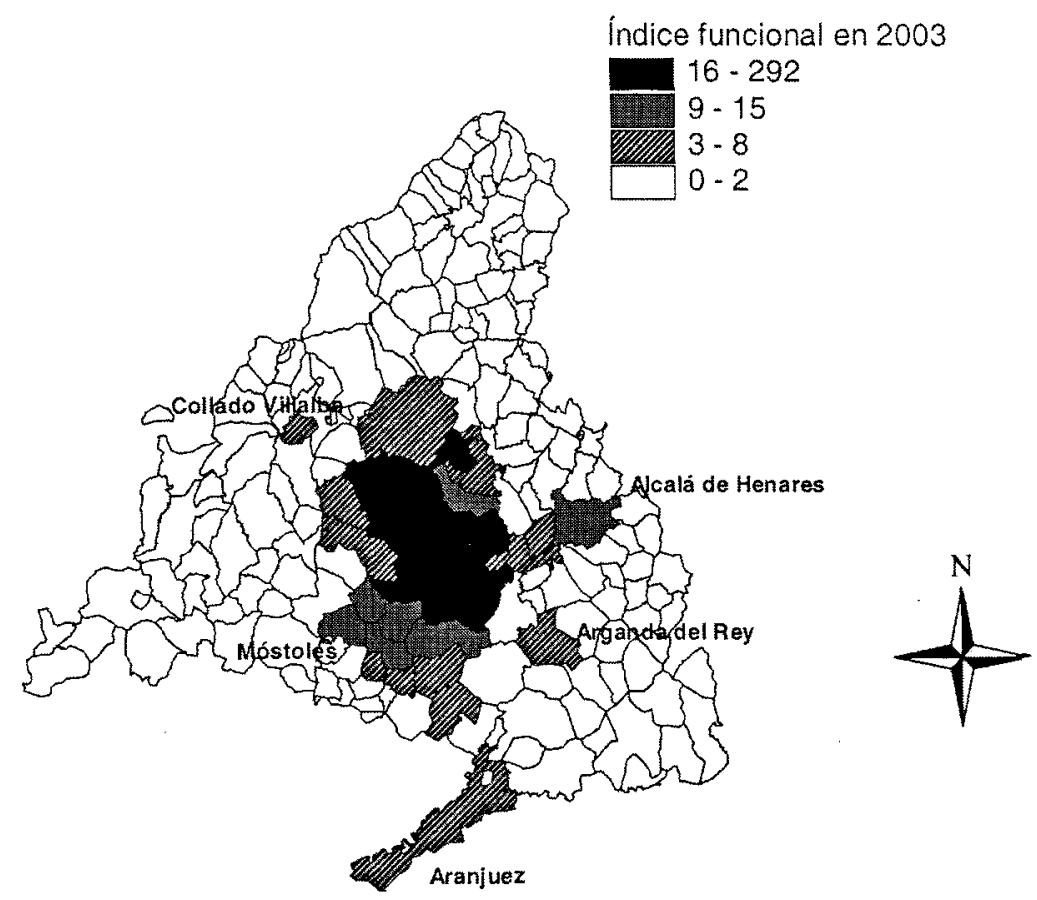

Mapa 3. Valores del índice funcional en los municipios de la Comunidad de Madrid. Fuente: Elaboración propia.

de Barajas. La calidad medioambiental, los valores paisajísticos, el diseño arquitectónico y urbanístico y la presencia de servicios avanzados para las empresas son elementos comunes.

El mapa $n^{\circ} 3$, elaborado con datos sobre comercios y servicios procedentes de los servicios estadísticos de la Comunidad de Madrid, muestra, a través de la representación cartográfica del índice funcional, el desigual peso de estas actividades en los municipios madrileños. Los valores del índice funcional expresan el desigual dinamismo de estos municipios, muy ligado a desarrollos urbanísticos y poblacionales en relación con su proximidad a la ciudad de Madrid y su ubicación en los principales ejes viarios. Pero también son un claro reflejo de las acciones promovidas por el planeamiento regional al que se ha hecho anteriormente referencia. La expansión de los altos valores del índice funcional hacia los bordes de la región revela, a su vez, las crecientes demandas de suelo por parte de actividades centrales que buscan nuevas localizaciones dentro de una aglomeración consolidada como metrópoli internacional en la red mundial de ciudades. 


\section{Conclusión: hacia un modelo territorial menos centralizado}

En los comienzos del tercer milenio, las lógicas espaciales de la globalización se materializan en una forma urbana difusa que desborda la antigua área metropolitana de Madrid y se extiende más allá de la Comunidad, siempre siguiendo las vías principales de comunicación. El modelo metropolitano de los años 60 , basado en la contraposición entre unas áreas centrales muy congestionadas e indiferenciadas y una periferia industrial y residencial, con servicios insuficientes e infraestructuras inadecuadas para la población y el aparato productivo, está más que superado.

Hasta un pasado reciente, más allá del área metropolitana configurada desde 1963 por COPLACO, se extendían las áreas propiamente rurales, caracterizadas por el vaciamiento demográfico y el abandono de las actividades agrarias. Hoy, la creciente demanda de suelos especializados, residenciales, industriales, comerciales y de oficinas para satisfacer las exigencias de la globalización, se extiende por toda la Comunidad. Los límites entre el campo y la ciudad desaparecen y los usos urbanos del suelo aumentan su intensidad conforme se reduce la distancia al centro de la aglomeración, junto a las carreteras principales y en todas aquellas localizaciones que se benefician de las acciones de ordenación del territorio planificadas por la administración.

Las estrategias de reequilibrio territorial se dejan sentir cada vez más en la evolución de la tradicional estructura radial de nuestra región hacia una estructura más reticular. Las operaciones acometidas han generado condiciones objetivas de desarrollo que permiten a la Comunidad de Madrid alcanzar niveles mayores de competitividad con otras regiones, no sólo del sur de Europa sino del conjunto del planeta. Todo esto es aún más cierto si se enmarca en un contexto en el que se observa como las lógicas de la globalización imponen un funcionamiento mundial de las ciudades en red. Como señala M. Castells (1997), la eficacia de los flujos se antepone en la actualidad a la de los lugares y las ciudades han de ser consideradas como estructuras complejas que resultan de capas superpuestas de organización del territorio según variables de distinta naturaleza.

En la región madrileña, los espacios productivos de última generación y los de centralidad y ocio más recientes, en localizaciones periféricas del área metropolitana y fuera de ella, impulsan la creación de áreas residenciales que, aparte de resolver necesidades primordiales de alojamiento, han de servir para acortar desplazamientos entre lugares de trabajo y de residencia, y para paliar sus costes económicos y sociales. En este sentido, resulta más que significativo el caso de Arroyomolinos, municipio a 23 kilómetros de la capital, donde se ha instalado el parque comercial y de ocio "Xanadú". Este parque, con la pista cubierta de esquí más grande de Europa y un importante complejo comercial y de servicios junto a él, se ha convertido en un 
nuevo elemento de centralidad en el sur de la Comunidad de Madrid. Su puesta en funcionamiento ha permitido la creación de 4.000 empleos directos y 6.000 indirectos. Como consecuencia de ello, la población residente del municipio ha aumentado espectacularmente y lo seguirá haciendo en los próximos años. El municipio de Arroyomolinos, con menos de 300 habitantes en los años 80, en 2003 contaba con unos 3.000 y, una vez estén terminadas las 10.200 viviendas previstas por su Plan General de Ordenación Urbana, alcanzará más de 30.000 vecinos.

Dentro del modelo de "ciudad regional" difusa que está naciendo en Madrid y que rebasa funcionalmente los límites de su Comunidad, la mejora de las infraestructuras de transportes está siendo una pieza determinante. El cierre de las vías rápidas de circunvalación exteriores a la M-30, las M-45 y M-50, y la construcción de autopistas radiales de peaje, paralelas a las actuales carreteras nacionales, garantizan la eficacia del modelo territorial descentralizado y polinuclear que los agentes sociales, políticos y económicos impulsan. Especialmente importante por sus efectos urbanizadores y vertebradores del territorio está siendo la ampliación de la red de metro, dentro de la capital y fuera del municipio madrileño, primero con la prolongación de la línea 9 hasta Arganda del Rey, y desde el 11 de abril de 2003, con la inauguración de "metrosur". Esta línea, la número 12 de la red, con un trazado circular de 40,5 kilómetros de longitud y 28 estaciones, une los municipios de Alcorcón, Leganés, Fuenlabrada, Getafe y Móstoles, prestando servicio a un millón de personas. La conexión con el resto de la red del metropolitano se realiza a través de la prolongación de la línea 10 y de varios enlaces con las cercanías de RENFE. A principios de julio de 2004, se ha decidido mejorar estas conexiones aprovechando el acuerdo entre la administración autonómica y el ayuntamiento de Getafe para llevar a esta localidad la prolongación recientemente prevista de la línea 3 del metropolitano.

Para la presente legislatura, 2003-2007, el gobierno regional ha propuesto un ambicioso programa de ampliación del metropolitano y de implantación de metro ligero en conexión con el anterior, dentro de lo que se denominaría "metronorte" y "metroeste". Mediante estas actuaciones se quiere mejorar las comunicaciones de la capital con el norte metropolitano, el noroeste y el corredor del Henares. "Metronorte" proporcionará también servicio a los PAUs de la periferia norte (las Tablas, Sanchinarro y Montecarmelo) y a la proyectada prolongación de la Castellana. De ese modo, se atendería a miles de usuarios potenciales (340.000 en el norte y 382.000 en el corredor del Henares), y se evitaría el colapso de tráfico que se producirá, de no existir esta alternativa, una vez que se encuentren terminados los desarrollos urbanísticos de la aglomeración madrileña en vías de ejecución.

En definitiva, las lógicas espaciales de la globalización y las respuestas que los agentes económicos, sociales y políticos de la región madrileña les están dando, están siendo responsables de las transformaciones territoriales y paisajísticas más importantes y rápidas experimentadas por la región madrileña a lo largo de toda su histo- 
ria. A la vez, los propios mecanismos de la globalización están favoreciendo el aumento de las desigualdades ante el empleo estable, las dificultades en el acceso a la vivienda en propiedad y una inmigración extranjera masiva que en ciertas zonas y barrios alcanza ya a más del $50 \%$ de sus efectivos demográficos. Todo ello obliga a los responsables de la gestión política y a los planificadores a avanzar en la aplicación de estrategias de ordenación del territorio y de mejora social. Se trata no sólo de corregir desequilibrios y de asegurar la eficacia de los agentes económicos, sino de hacer real el desarrollo sostenible de la región, conservar el patrimonio cultural, responder a las necesidades de una población que envejece y a los riesgos de exclusión y marginación social que surgen. Para la observación y tratamientos de todos estos aspectos, estrechamente ligados a las lógicas de la globalización, la "Región urbana" madrileña o, si se prefiere, la "Ciudad-región" de Madrid, se configura como un laboratorio privilegiado y un muestrario de problemáticas territoriales y sociales que alcanzan las dimensiones globales y universales que corresponden a un espacio que, también en su conjunto, nadie duda de calificar como "Ciudad global".

\section{Bibliografía}

Benko, G. et Lipietz, A. (dirs.) (2000): La richesse des régions, París, PUF.

Boyer, J. C. (2003): Les villes europénnes. Ed. Hachette, Paris.

Borja, J. y Castells, M. (1998): Local y global. La gestión de las ciudades en la era de la información. Madrid, UNCHS-Taurus.

Blanco Fernández, J. (2004): La emergencia de las nuevas ciudades en la Era Global. Gijón, Ed. Trea.

Castells, M. (1997): La Ciudad Informacional. Tecnologias de la información y proceso urbano-regional. Madrid, Ed. Alianza.

COMITÉ DE DESARROLLO TERRITORIAL, UNIÓN EUROPEA (1999): Estrategia Territorial Europea. Hacia un desarrollo equilibrado y sostenible de la Unión Europea. Luxemburgo.
Clark, D. (1999, $2^{\text {a }}$ ed.): Urban World / Global City. London and New York, Ed. Routledge.

Córdoba Ordóñez, J. y Gago García, C. (2002): "Madrid en el escenario de un sistema mundial de ciudades". En "Madrid, punto de encuentro" Anales de Geografia de la Universidad Complutense, Homenaje a J. María Sanz García, vol. Extraordinario, p. 203-219.

Gervais-Lambony, M. A. (dir.) (2001): Les très grandes villes. Neuilly, Éditions Atlande.

Graham, S. and Marvin, S. (2001): Splintering Urbanism. London, Routledge.

Hall, P. (1998): Cities and Civilization. New York, Pantheon.

Maillat, D. et Grosjean, N. (1999): "Globalisation et systèmes territoriaux de production". Working Paper, IRER, no $9906 \mathrm{a}$, Université de Neuchatel, 17 pp. 
Molina Ibáñez, M. (2002): "Madrid, metrópoli global". En Madrid, punto de encuentro, Anales de Geografia de la Universidad Complutense, Homenaje a J. María Sanz García, vol. Extraordinario, p. 349-356.

PLAN GENERAL DE MADRID DE 1997 (1997): Área de planeamiento específico (A.P.E.).

PLAN REGIONAL DE ESTRATEGIA TERRITORIAL. BASES (1995): CAM, Madrid.

Pont Vidal, J. (2004): Los ciudadanos se movilizan: los movimientos sociales. Los movimientos sociales y la globalización en España. Barcelona, Flor del Viento Ediciones.

Rozenblat, C. et Cicille, P. (2003): Les villes européennes. Analyse comparative. Paris, La Documentation Française, Datar, Avril, 94 pp.

Ruíz Gallardón, A. (1999): "Ganar el futuro". En García Delgado, J. L. Director, Estructura económica de Madrid, Madrid, Civitas.

Sassen, S (1998): "Ciudades en la economía mundial: enfoques teóricos y metodológi- cos" Rev. Eure. Vol. XXIV, n ${ }^{\circ} 71$, marzo, p. 5-25.

Smith, M. P. (2001): Transnational Urbanism. Locationg Globalization. Massachusetts (USA), Oxford (UK), Ed, Blackwell Publishers, Malden.

Soja, E.W. (2000): Posmetropolis: critical studies of cities and regions. Blackwell, Oxford.

Veltz, P. (1999): Mundialización, ciudades y territorio. Madrid, Ed. Ariel

Webster, F. (1999): "Information and Communications Technologies: Luddism Revisited". En Technocities. Ed, by Downey, J. \& Mcguigan, J., Sage, 1999.

Zárate Martín, M. A. (2000): "Toledo, Ciudad Patrimonio de la Humanidad". Boletín de la Real Sociedad Geográfica, tomo CXXXVI, p. 137-184.

Zárate Martín, M. A. (2003): Efectos de la Globalización en la región urbana de Madrid. Madrid, Ed. UNED. 